\title{
SIRT3: A New Regulator of Cardiovascular Diseases
}

\author{
Wei Sun, ${ }^{1}$ Caixia Liu, ${ }^{2}$ Qiuhui Chen, ${ }^{3}$ Ning Liu, ${ }^{1}$ Youyou Yan, ${ }^{1}$ and Bin Liu ${ }^{1}{ }^{1}$ \\ ${ }^{1}$ Department of Cardiology, The Second Hospital of Jilin University, 218 Ziqiang Road, Changchun 130041, China \\ ${ }^{2}$ Department of Neurology, The Liaoning Province People's Hospital, 33 Wenyi Road, Shenyang 110016, China \\ ${ }^{3}$ Department of Neurology, The Second Hospital of Jilin University, 218 Ziqiang Road, Changchun 130041, China
}

Correspondence should be addressed to Bin Liu; liubin3333@vip.sina.com

Received 1 November 2017; Revised 20 December 2017; Accepted 4 January 2018; Published 13 February 2018

Academic Editor: Ada Popolo

Copyright (c) 2018 Wei Sun et al. This is an open access article distributed under the Creative Commons Attribution License, which permits unrestricted use, distribution, and reproduction in any medium, provided the original work is properly cited.

\begin{abstract}
Cardiovascular diseases (CVDs) are the leading causes of death worldwide, and defects in mitochondrial function contribute largely to the occurrence of CVDs. Recent studies suggest that sirtuin 3 (SIRT3), the mitochondrial NAD ${ }^{+}$-dependent deacetylase, may regulate mitochondrial function and biosynthetic pathways such as glucose and fatty acid metabolism and the tricarboxylic acid (TCA) cycle, oxidative stress, and apoptosis by reversible protein lysine deacetylation. SIRT3 regulates glucose and lipid metabolism and maintains myocardial ATP levels, which protects the heart from metabolic disturbances. SIRT3 can also protect cardiomyocytes from oxidative stress-mediated cell damage and block the development of cardiac hypertrophy. Recent reports show that SIRT3 is involved in the protection of several heart diseases. This review discusses the progress in SIRT3-related research and the role of SIRT3 in the prevention and treatment of CVDs.
\end{abstract}

\section{Introduction}

Cardiovascular diseases (CVDs) are the leading causes of death worldwide, with $>80 \%$ CVD-related deaths in lowand middle-income countries. Globally, the incidence of CVD-related deaths increased by $14.5 \%$ (95\% confidence interval (95\%CI): $12.1 \%-17.1 \%$ ) between 2006 and 2016, although age-standardized death rates due to CVD decreased by $14.5 \%$ (95\%CI: $12.5 \%-16.2 \%)$ over this time period [1]. By 2030, almost 23.6 million people are predicted to die from CVDs, mainly from heart disease and stroke $[2,3]$. The molecular mechanisms of CVD include accumulation of reactive oxygen species (ROS), imbalance of vasoconstriction/vasodilation, chronic inflammation, and premature senescence, which are closely related to sirtuin-mediated regulation [4].

Sirtuins are a family of nicotinamide adenine dinucleotide- $\left(\mathrm{NAD}^{+}-\right)$dependent histone deacetylases, which are highly conserved across species from bacteria to humans [5-7]. It was first discovered in yeast as "silent mating type information regulator 2" (SIR2) in 1979 [8]. In mammals, seven SIR2 homologs (SIRT1-7) were identified, which differ in subcellular localization and mechanisms of biological regulation. SIRT3 is located in the mitochondrial matrix [9]; however, several studies suggest that it is also located in the nucleus and cytoplasm [10-12]. SIRT3 is a deacetylase that regulates the majority of mitochondrial lysine acetylation [13-15]. Recent studies show that SIRT3 plays important roles in cardiovascular physiology and pathology and describe the mechanisms via which SIRT3 regulates cardiac processes [16-25]. In this review, we will discuss current opinions on the role of SIRT3 in CVDs and speculate on its prospects for clinical application.

\section{SIRT3: Function and Targets}

2.1. SIRT3 and Energy Metabolism. More than $90 \%$ of ATP in the normal myocardium is derived from mitochondrial oxidative phosphorylation, followed by anaerobic glycolysis of glucose. Fatty acid beta oxidation is the main source of mitochondrial oxidative phosphorylation, followed by glucose, lactic acid, and ketone body aerobic oxidation [26]. SIRT3 regulates the enzymatic activity of the key enzymes of 
oxidative phosphorylation via deacetylation, thereby regulating mitochondrial energy metabolism (Table 1).

Reduced SIRT3 levels promote glycolysis via two mechanisms. First, the peptidylprolyl isomerase D (cyclophilin D) is in a highly acetylated state in the absence of SIRT3, which activates hexokinase II (HK2), a key glycolytic enzyme in the mitochondrial outer membrane. HK2 phosphorylates glucose to produce glucose-6-phosphate (G6P), the first step in most glucose metabolism pathways [27, 28]. Second, loss of Sirt3 increases ROS production, which stabilizes hypoxiainducible factor- (HIF-) $1 \alpha$, a transcription factor that regulates glycolytic gene expression $[29,30]$.

SIRT3-mediated deacetylation modifies and activates long-chain acyl-CoA dehydrogenase (LCAD), which is the key enzyme of fatty acid $\beta$ oxidation, to promote fatty acid metabolism. Compared to wild-type mice, Sirt3 knockout (Sirt3-KO) mice exhibit hallmarks of fatty acid oxidation disorders during fasting, including reduced ATP levels and intolerance to cold exposure [31]. In addition, SIRT3 can regulate other enzymes of fatty acid oxidation, such as medium chain-specific acyl-CoA dehydrogenase (ACADM) and acylglycerol kinase (AGK) [32]. Acyl-CoA synthetase short-chain family member 2 (ACSS2) plays a key role in lipogenesis by converting acetate to acetyl-CoA, which enters the tricarboxylic acid cycle (TCA) to promote oxidative phosphorylation and produce energy. Previous studies show that SIRT3 deacetylates and activates ACSS2 [33, 34]. In addition, SIRT3 regulates ketone body production by deacetylating and activating 3-hydroxy-3-methylglutaryl-CoA synthase 2 (HMGCS2), which is the rate-limiting enzyme in ketone-body biosynthesis $[35,36]$.

Most amino acids are decomposed by aminotransferasemediated transfer of an $\alpha$-amino moiety to $\alpha$-ketoglutarate to form glutamate. SIRT3-mediated deacetylation activates glutamate dehydrogenase 1 (GLUD1), the enzyme that produces $\alpha$-ketoglutarate from glutamate and is involved in both TCA cycle and ammonia metabolism [15]. SIRT3 can also upregulate the urea cycle (UC) through deacetylation and activation of ornithine transcarbamylase (OTC), the key enzyme of UC, suggesting that SIRT3 increases amino acid catabolism and ammonia detoxification during periods of metabolic stress [37]. Furthermore, SIRT3 regulates mitochondrial protein synthesis by deacetylation of the ribosomal protein MRPL10 [38].

The TCA cycle and electron transport chain (ETC) couple redox balance with ATP generation [39]. SIRT3-mediated deacetylation activates the components of the ETC complex, including NDUFA9 (complex I) [40] and SDHA (complex II) [41, 42]. SIRT3 also regulates ATP synthase activity [43]. Moreover, SIRT3 deacetylates IDH2, a key enzyme of TCA, which promotes oxidation of isocitrate to $\alpha$-ketoglutarate and produces NADPH [44].

2.2. SIRT3 and Oxidative Stress. Oxidative stress causes extensive accumulation of intracellular ROS; destruction of proteins, lipids, and nucleic acids; membrane phospholipid peroxidation; and mitochondrial DNA mutations, which lead to severe cell damage and death. It is closely related to cardiac hypertrophy $[17,45-47]$, coronary atherosclerosis
$[48,49]$, hyperlipidemia $[37,50]$, diabetes $[51,52]$, and other diseases. The mitochondrial ETC is the main source of ROS, and SIRT3 enhances the ability of the mitochondria to cope with ROS in multiple ways. The key superoxide scavenger, Mn superoxide dismutase (SOD2), can reduce superoxide production and protect against oxidative stress. SIRT3 directly regulates the activity of SOD2 by deacetylation [53-57]. Moreover, SIRT3 can sequester forkhead box O3a (FOXO3A) in the nucleus to increase the transcription of SOD2 and other key genes involved in antioxidation. Human serum FOXO3A and SIRT3 can be used as markers for ageing, with therapeutic potential for maintenance of healthy ageing $[17,58]$. Second, SIRT3 deacetylates and activates the TCA cycle enzyme IDH2 and helps replenish the mitochondrial pool of NADPH [44]. NADPH is a key reducing factor that affects glutathione reductase, a part of the antioxidant defense system against cellular oxidative stress [44]. In addition, SIRT3 promotes effective electron transport via deacetylation of ETC complex components, which indirectly reduces ROS production [57].

2.3. SIRT3 and Apoptosis. Currently, the relationship between SIRT3 and apoptosis is not clear, and whether SIRT3 promotes or inhibits apoptosis is still controversial. However, SIRT3 was shown to mainly inhibit cardiomyocyte apoptosis in most studies. As mentioned previously, SIRT3 can inhibit apoptosis by regulating oxidative stress. In addition, SIRT3 also inhibits apoptosis by the following route: first, SIRT3 deacetylates and activates optic atrophy 1 (OPA1). Loss of OPA1 impairs mitochondrial fusion, perturbs cristae structure, and increases the susceptibility of cells toward apoptosis [59, 60]. Second, SIRT3 activates Ku70 by deacetylation. The activated Ku70 binds to Bax, which inhibits Bax-induced cardiomyocyte apoptosis [58]. Third, SIRT3 deacetylates cyclophilin D and closes the mitochondrial permeability transition pore (mPTP) to maintain the normal morphology of mitochondria, thereby inhibiting apoptosis $[45,61,62]$.

\section{SIRT3 in Cardiovascular Disease}

3.1. SIRT3 in Ischemic Heart Disease. Ischemic heart disease (IHD) and cerebrovascular disease (stroke) together accounted for more than $85.1 \%$ of all CVD deaths in 2016. Total deaths from IHD increased by $19.0 \%$ (95\%CI: $16.2 \%-$ $22.1 \%$ ), which contributed largely to the overall increase in total deaths from CVD in 2016 [1]. Severe ischemia can lead to a variety of pathological processes, including metabolic disorders and myocardial cell ultrastructure damage, which can be catastrophic for myocardial cells [63].

SIRT3 downregulation increases the susceptibility of cardiac-derived cells and adult hearts to ischemia-reperfusion (IR) injury and may contribute to a higher level of IR injury in the aged heart $[22,64]$. SIRT3 knockout leads to coronary microvascular dysfunction and impairs cardiac recovery post myocardial ischemia $[18,65]$. Klishadi et al. reported that SIRT3 levels decreased after IR induced by left anterior descending artery occlusion [66]. The renin-angiotensinaldosterone system (RAAS) is involved in ischemic injury 
TABLE 1: Known targets of SIRT3 and function.

\begin{tabular}{|c|c|c|c|}
\hline Function & Gene symbol & Gene name & References \\
\hline \multicolumn{4}{|l|}{ Energy metabolism } \\
\hline Glycolysis & PPID & Peptidylprolyl isomerase D (cyclophilin D) & {$[27]$} \\
\hline Fatty acid oxidation & ACADL & Long-chain Acyl-CoA dehydrogenase (LCAD) & {$[31]$} \\
\hline Ketone body synthesis & HMGCS2 & 3-Hydroxy-3-methylglutaryl-CoA synthase 2 , mitochondrial & {$[35]$} \\
\hline Acetate metabolism & ACSS2 & Acyl-CoA synthetase short-chain family member 2 & {$[33,34]$} \\
\hline Urea cycle & OTC & Ornithine transcarbamylase & [37] \\
\hline Amino acid catabolism & GLUD1 & Glutamate dehydrogenase 1 (GDH) & {$[15]$} \\
\hline Mitochondrial protein synthesis & MRPL10 & Mitochondrial ribosomal protein L10 & {$[38]$} \\
\hline \multirow{3}{*}{ Oxidative phosphorylation } & NDUFA9 & NADH dehydrogenase (ubiquinone) $1 \alpha$ subcomplex 9 & {$[40]$} \\
\hline & SDHA & Succinate dehydrogenase complex, subunit A, flavoprotein & {$[41,42]$} \\
\hline & ATP5a & $\mathrm{F}_{1} \mathrm{~F}_{0}-\mathrm{ATPase}$ subunit $\alpha$ & {$[43]$} \\
\hline TCA cycle & IDH2 & Isocitrate dehydrogenase 2 , mitochondrial & {$[44]$} \\
\hline \multicolumn{4}{|l|}{ Oxidative stress } \\
\hline Transcriptional activation & FOXO3a & Forkhead box O3a & {$[17,58]$} \\
\hline ROS & SOD2 & Superoxide dismutase 2 , mitochondrial (MnSOD) & [53-57] \\
\hline \multirow{2}{*}{ Apoptosis } & OPA1 & Optic atrophy 1 & {$[59,60]$} \\
\hline & XRCC6 & $\mathrm{X}$-ray repair cross-complementing $6(\mathrm{Ku} 70)$ & [58] \\
\hline
\end{tabular}

[67, 68]. Angiotensin II downregulates SIRT3 [69], and drugs directly inhibiting RAAS normalized SIRT3 levels in the ischemic myocardium and improved cardiac function [40, $66,70]$. However, data on the instant effect of RAAS on SIRT3 during the acute ischemia is not available.

Atherosclerosis caused by vascular inflammation is also one of the pathophysiological mechanisms of IHD. Many studies have shown that SIRT3 is involved in the regulation of vascular inflammation. Trimethylamine-N-oxide (TMAO) activates nucleotide-binding oligomerization domain-like receptor family pyrin domain containing 3 (NLRP3) inflammasomes to induce vascular inflammation, which promotes atherosclerosis. TMAO was found to promote ROS generation, especially mitochondrial ROS, and inhibit SOD2 activation and SIRT3 expression in HUVECs and aortas from $\mathrm{ApoE}^{-1-}$ mice. Overexpression of SIRT3 resulted in protection against TMAO-induced activation of NLRP3 inflammasomes in endothelial cells. TMAO can also not further inhibit SOD2 and activate NLRP3 inflammasome in SIRT3 siRNAtreated HUVECs and aortas from SIRT3 ${ }^{-/-}$mice [71].

Moreover, a study of aerobic interval training showed that compared to the control group, Sirt3 expression was reduced in the myocardium of rats with acute myocardial injury, while the interval of aerobic breathing resulted in increased Sirt3 expression and protected against myocardial infarction-induced oxidative injury [72]. However, Winnik et al. observed that SIRT3 knockout did not affect the progression of atherosclerotic lesions and plaque stability [73]. Therefore, the role of SIRT3 in atherosclerosis remains to be elucidated.

3.2. SIRT3 in Hypertrophy and Heart Failure. Hypertrophy is a compensatory response that results in cardiomyocyte death, fibrosis, and cardiac pressure or volume overloadinduced heart failure because of structural changes in myocardial cells [74]. An increasing number of studies revealed that poor SIRT3 activity is one of the causes of cardiac hypertrophy and heart failure $[17,45,75]$.

Four weeks after a transverse aortic constriction, the ejection fraction in Sirt3-KO mice was lesser than that in wildtype mice, which was accompanied by an increase in cardiac hypertrophy and fibrosis $[19,76]$. Sirt3-KO mice showed a decreasing trend for palmitate oxidation, glucose oxidation, oxygen consumption, respiratory capacity, and ATP synthesis, whereas glycolytic rates were increased [19]. Sirt3-KO mice also showed abnormal lipid accumulation [77]. SIRT3 levels are reduced, and mitochondrial protein lysine acetylation is elevated in models of hypertensive heart failure, suggestive of impaired SIRT3 activity [78]. The low SIRT3 levels may be associated with the downregulation of PGC1a [79]. Moreover, as a DNA repair enzyme, poly(ADPribose)-polymerase 1 (PARP-1) also uses $\mathrm{NAD}^{+}$as a cosubstrate [80]. Overactivation of PARP-1 in failing hearts increases the competition between SIRT3 and $\mathrm{NAD}^{+}$, which leads to large-scale consumption of cellular $\mathrm{NAD}^{+}$and a decrease in SIRT3 activity [80, 81]. In addition, RIP140, which is involved in the pathogenesis of cardiac hypertrophy and heart failure, also inhibits SIRT3 [82].

Recent studies show that SIRT3 regulates energy metabolism [30, 32], resists oxidative stress [17, 45, 47, 58], and prevents cardiac hypertrophy. Nicotinamide mononucleotide adenylyltransferase 3 (NMNAT3) is the only known enzyme of the $\mathrm{NAD}^{+}$synthesis pathway that is localized in the mitochondrial matrix [83]. NMNAT3 is a SIRT3 substrate, deacetylation of which enhances its activity. Subsequently, NMNAT3 contributes to SIRT3-mediated antihypertrophic effects in cardiomyocytes by supplying $\mathrm{NAD}^{+}[84,85]$. Moreover, a study showed that SIRT3-LKB1-AMPK pathway activation improved cardiac hemodynamics and preserved the ejection fraction $[25,86]$. SIRT3 can also protect against 
cardiac fibrosis by inhibiting myofibroblast transdifferentiation via the STAT3-NFATc2 [87] and $\beta$-catenin/PPAR- $\gamma$ [88] signaling pathways.

In addition, emerging evidence indicates that impaired angiogenesis may contribute to hypertension-induced cardiac remodeling. A mouse myocardial fibrosis model induced by angiotensin II confirmed that SIRT3 enhanced mitochondrial autophagy, mediated by Pink/Parkin, and attenuated the production of mitochondrial ROS, restored vascular budding and tube formation, and improved myocardial fibrosis and cardiac function [89].

3.3. SIRT3 in Drug-Induced Cardiotoxicity. Drug-induced cardiotoxicity is now a common clinical practice. In particular, chemotherapeutic drug-induced cardiotoxicity has spawned the emergence of the interdisciplinary field of cardio-oncology. Recently, practice guidelines were published to guide clinical work in this interdisciplinary area [90].

Anthracycline chemotherapeutics such as doxorubicin (DOX) are commonly used for the clinical treatment of tumors. DOX induces cardiotoxicity via mitochondrial dysfunction [91]. A study showed that DOX reduced SIRT3 expression and correspondingly increased mitochondrial protein acetylation, an effect that was more pronounced in SIRT3-KO mice [23, 92, 93]. SIRT3 overexpression deacetylates and activates OPA1, which regulates mitochondrial dynamics and protects cardiomyocytes from doxorubicininduced cardiotoxicity [23, 60, 93, 94]. Moreover, SIRT3 may also attenuate doxorubicin-induced cardiac hypertrophy and mitochondrial dysfunction via suppression of BNIP3 [95].

3.4. SIRT3 in Diabetic Cardiomyopathy and Cardiac Lipotoxicity. Diabetes and obesity are important risk factors for CVD. Studies show that the mitochondrial sirtuin family is involved in insulin resistance with diabetes mellitus $[19,20,24]$.

SIRT3 can prevent and even reverse diabetes-induced retinal [96-98], skeletal [99-101], and cardiac damage [102, 103]. The SIRT3-FOXO3A-Parkin signaling pathway may play a vital role in the development of diabetic cardiomyopathy [102]. Melatonin protects against diabetic cardiomyopathy through MST1/SIRT3 signaling [104], and garlic protects patients with diabetic cardiomyopathy from oxidative stress by enhancing SIRT3 activity [105]. Moreover, $A P L N$ gene therapy increases angiogenesis and improves cardiac functional recovery in diabetic cardiomyopathy via upregulation of the SIRT3 pathway [103].

SIRT3 levels were low in the hearts of high-fat diet(HFD-) fed obese mice, and cardiac lipotoxicity was high in Sirt3 knockout mice [19, 20, 24]. SIRT3 possibly regulates lipotoxicity by promoting lipid metabolism, reducing fatty acid accumulation and oxidative stress injury $[31,32,106]$, and restoring cardiac remodeling function [20,24].

Oxidative stress is the common pathophysiology of diabetic cardiomyopathy and cardiac lipotoxicity [107, 108]. SIRT3 protects pancreatic beta cells from lipotoxicity by antagonizing oxidative stress-induced cell damage [109].
Thus, SIRT3 can inhibit diabetic cardiomyopathy and cardiac lipotoxicity.

3.5. SIRT3 in Hypertension. Hypertension, a common risk factor for diseases, such as cardiovascular, cerebrovascular, and kidney disease, has become a major risk factor for premature death and disability worldwide [110]. The roles of SIRT3 in hypertension are well documented. Waypa et al. first demonstrated that Sirt3 deletion does not augment hypoxia-induced ROS signaling or its consequences in the cytosol of pulmonary arterial smooth muscle cells, nor the development of pulmonary arterial hypertension (PAH) [111]. However, Paulin et al. more recently reported that SIRT3 is downregulated in a rat PAH model and in human PAH tissues. SIRT3 ${ }^{-1-}$ and SIRT3 ${ }^{+-}$mice develop PAH in a gene dose-dependent manner. Sirt3 gene therapy even reverses PAH in rats in vivo and human vascular cells in vitro [30]. Recently, Dikalova et al. showed that downexpression and redox inactivation of Sirt3 lead to SOD2 inactivation and contribute to the pathogenesis of hypertension [55]. These data suggest that Sirt3 responses may be cell type specific or restricted to certain genetic backgrounds.

\section{Therapeutic Application}

SIRT3 plays an important role in the development and progression of CVDs. Therefore, promotion of SIRT3 expression and activity via pharmacological pathways can delay the development of CVDs (Table 2).

4.1. Traditional Chinese Medicine. Components extracted from traditional Chinese medicine promoted SIRT3mediated cardioprotective effects [112]. Resveratrol, which is abundant in grapes and blueberries, is the first compound that was shown to activate SIRT3 $[113,114]$. A study demonstrated that resveratrol activates SIRT3 and downregulates the TGF- $\beta /$ Smad3 pathway to improve myocardial fibrosis [115]. Polydatin, a monocrystalline and a polyphenolic drug extracted from the traditional Chinese herb Polygonum cuspidatum, protects cardiomyocytes against myocardial infarction injury by upregulating autophagy and improving mitochondrial biogenesis via SIRT3 activity [116]. Berberine decreases DOX-induced cardiotoxicity by activating SIRT3 [117]. Honokiol, a natural biphenolic compound derived from the bark of magnolia trees, blocks and reverses cardiac hypertrophy by activating SIRT3 $[76,118]$. Pomegraniin A activates SOD2 through SIRT3-mediated deacetylation and reduces intracellular ROS [119]. Oroxylin A (OA), which is derived from the root of Scutellaria baicalensis, was found to be a SIRT3 activator in an in vitro model of cardiac myocyte insulin resistance [120] and plays roles in preventing myocardial contractile function and improving myocardial fibrosis and heart failure [121]. Curcumin, a phenolic compound extracted from the natural herb turmeric, can activate the PGC- $1 \alpha /$ SIRT3 signaling pathway to protect against mitochondrial impairment, and it can also stimulate SIRT1 to have cardioprotective effects [122].

4.2. Small Molecule Activators of SIRT3. Inactivation of PIKFYVE, an evolutionarily conserved lipid kinase that regulates 
TABLE 2: Therapeutic application of SIRT3.

\begin{tabular}{|c|c|c|c|}
\hline Categories & Representatives & Mechanism & References \\
\hline \multirow{7}{*}{ Traditional Chinese medicine } & Resveratrol & $\begin{array}{c}\uparrow \text { SIRT3 } \\
\downarrow \text { TGF- } \beta / \text { Smad3 }\end{array}$ & {$[113-115]$} \\
\hline & Polydatin & $\uparrow$ SIRT3 & [116] \\
\hline & Berberine & 个SIRT3 & {$[117]$} \\
\hline & Honokiol & †SIRT3 & {$[76,118]$} \\
\hline & Pomegraniin A & 个SIRT3/SOD2 & {$[119]$} \\
\hline & Oroxylin A & $\uparrow$ SIRT3 & {$[120,121]$} \\
\hline & Curcumin & $\uparrow$ PGC- $1 \alpha /$ SIRT3 & [122] \\
\hline \multirow{8}{*}{$\begin{array}{l}\text { Small molecule activators } \\
\text { of SIRT3 }\end{array}$} & $\downarrow$ PIKFYVE & 个SIRT3 & {$[123]$} \\
\hline & Melatonin & 个SIRT3 & {$[79,104,124,125]$} \\
\hline & Adjudin & $\uparrow$ SIRT3 & {$[126]$} \\
\hline & Minocycline & $\begin{array}{c}\uparrow \text { SIRT3/PHD2 } \\
\downarrow \text { HIF- } 1 \alpha\end{array}$ & {$[127]$} \\
\hline & MIPEP & $\uparrow$ SIRT3 & {$[128]$} \\
\hline & Metformin & $\uparrow$ SIRT3 & [129] \\
\hline & NMNAT3 & $\uparrow \mathrm{NAD}^{+} / \mathrm{SIRT} 3$ & {$[84]$} \\
\hline & $\mathrm{C} 12$ & $\uparrow S I R T 3 / M n S O D$ & {$[138]$} \\
\hline \multirow{6}{*}{ Signaling pathways } & EphB2 signaling & $\uparrow$ SIRT3/MnSOD & [139] \\
\hline & cAMP/PKA signaling & $\uparrow$ SIRT3/OPA1 & [59] \\
\hline & AMPK & $\uparrow$ SIRT3 & {$[140]$} \\
\hline & SIRT1 & 个SIRT3 & [141] \\
\hline & PLGA-PNIPAM-NaB & 个SIRT3 & {$[142]$} \\
\hline & PGC- $1 \alpha-\mathrm{HO}-1$ & $\uparrow$ SIRT3 & [143] \\
\hline \multirow{4}{*}{ MicroRNAs } & MicroRNA-210 & $\downarrow$ SIRT3 & {$[144]$} \\
\hline & MicroRNA-92a & $\begin{array}{l}\downarrow \text { SIRT2 (Drosophila, homologous to human } \\
\text { SIRT2 and SIRT3) }\end{array}$ & [145] \\
\hline & MicroRNA28-5p & $\downarrow$ SIRT3 & [146] \\
\hline & MicroRNA-421 & $\downarrow$ SIRT3/FOXO3 & {$[147]$} \\
\hline
\end{tabular}

pleiotropic cellular functions, suppresses excessive mitochondrial ROS production and apoptosis through a SIRT3-dependent pathway in cardiomyoblasts [123]. Moreover, melatonin treatment alleviated cardiac dysfunction and ameliorated myocardial ischemia-reperfusion injury via SIRT3-dependent regulation of oxidative stress and apoptosis [79, 104, 124, 125]. Adjudin, which functions as an antispermatogenic agent, was found to attenuate oxidative stress and cellular senescence by activating SIRT3 [126]. Minocycline inhibited HIF- $1 \alpha$-mediated cellular responses and protected the blood-brain barrier and hypoxic brain injuries via activation of the SIRT3-PHD2 pathway. It implied that minocycline may have roles in IHD [127]. Mitochondrial intermediate peptidase (MIPEP), a mitochondrial signal peptidase (MtSPase), may promote the maintenance of mitochondrial quality during caloric restriction (CR), which extends lifespan and suppresses ageassociated pathophysiology in various animal models by activating SIRT3 [128]. Metformin, a common drug for diabetes treatment, was found to modulate the appearance of atherosclerosis and reduce vascular events by increasing SIRT3 expression [129]. SIRT3 activity is dependent on the levels of cellular $\mathrm{NAD}^{+}$and exogenous supplementation of $\mathrm{NAD}^{+}[78,130-134]$. Therefore, an increase in the levels of NMNAT3, the rate-limiting enzyme for mitochondrial $\mathrm{NAD}^{+}$biosynthesis, also enhances the myocardiumprotective role of SIRT3 [84]. Moreover, a variety of SIRT3specific small molecules is being developed [135-137]. A recent study identified a novel SIRT3 activator, 7-hydroxy3-(4'-methoxyphenyl) coumarin (C12), which binds to SIRT3 with high affinity, promotes deacetylation of MnSOD, and modulates mitochondrial protein acetylation and ROS [138].

4.3. Signaling Pathways. In addition, other signaling paths can also increase SIRT3 activity. EphB2 signaling-mediated SIRT3 expression promotes MnSOD-mediated mtROS scavenging [139]. Mitochondrial cAMP/PKA signaling controls SIRT3 levels and proteolytic processing of OPA1 [59]. AMPK attenuates the cardiac remodeling parameters and improves cardiac function via the SIRT3/oxidative stress signaling pathway [140]. SIRT1 activation induces SIRT3 and the combined functions of the nuclear-mitochondrial triad switch glycolysis to fatty acid oxidation and immunity from activation to repression in acute inflammation [141]. A study 
that is expected to be used in clinical trials showed that PLGA-PNIPAM microspheres loaded with the gastrointestinal nutrient $\mathrm{NaB}$ specifically binds to SIRT3 and activates its deacetylase function, thereby inhibiting ROS generation and autophagy, promoting angiogenesis and protecting cardiomyocytes in acute myocardial infarction (AMI) [142]. The peroxisome proliferator-activated receptor gamma coactivator 1-alpha- (PGC-1 $\alpha$-) heme oxygenase- (HO-1-) SIRT3 pathway increases mitochondrial viability and provides metabolic protection [143].

4.4. MicroRNAs. MicroRNAs are single-stranded, noncoding small molecule RNAs that inhibit translation or induce target molecule degradation. Our previous study found that microRNA-210 can indirectly regulate the expression and activity of SIRT3 and promote cellular energy metabolism [144]. A study of Drosophila circadian rhythms found that microRNA-92a modulates neuronal excitability by suppressing the expression of SIRT2, which is homologous to human SIRT2 and SIRT3 [145]. Poulsen et al. found that high glucose levels coupled with oxidative stress resulted in the upregulation of microRNA28-5p, which directly inhibited expression of SIRT3 [146]. To date, the only microRNA for which SIRT3 was identified as a functionally relevant target was microRNA-421. MicroRNA-421 acts upstream of the SIRT3/FOXO3 pathway to modulate oxidant stress and lipid metabolism [147]. Although the study of the regulation of SIRT3 by microRNAs has only just begun, it may provide a new approach for the application of SIRT3.

\section{Conclusion and Outlook}

An important pathophysiological mechanism of cardiovascular disease is mitochondrial damage and dysfunction. As shown in Table 1, SIRT3 plays important roles in cellular energy metabolism, oxidative stress, and apoptosis. Moreover, it can potentially be used for protection against CVDs. Currently, small molecule drug screening for SIRT3 constitutes an important research direction. We believe that, in the near future, studies on SIRT3 will generate new approaches for the prevention and treatment of CVDs. SIRT3 can also regulate systemic inflammation, improving the whole body's metabolic balance. Although much progress has been made in the study of SIRT3, there are still many problems to be clarified: (1) the sirtuin family plays an important role in cardiovascular regulation; however, the physiological and pathological effects of SIRT4 and SIRT5 are unclear. (2) Sirtuins are the sentries of mitochondrial homeostasis; however, the interactomes of mitochondrial sirtuins are quite different from each other, which suggests that there is a diversity and complexity of sirtuin functions within the mitochondrion. A better understanding of the mechanism of mitochondrial regulation of CVD will be further advanced by studies of the regulatory networks acting among the sirtuin family members. (3) SIRT3, named "the longevity gene," is closely related to the ageing of the human body. However, there is currently little data on the function of SIRT3 in ageing-related decline in cardiovascular functions. Further research to address these questions will be helpful in advancing our understanding of the roles of SIRT3 in CVD in order to facilitate the development of clinical diagnostic therapies.

\section{Conflicts of Interest}

The authors declare that they have no conflict of interests.

\section{Authors' Contributions}

Wei Sun and Caixia Liu contributed equally to this work.

\section{Acknowledgments}

This study was supported by grants from the National Natural Science Foundation of China (no. 81570250), the Ph.D. Outstanding Personnel Development Program of Bethune Medical Department (yb201503), and the National Clinical Key Specialty Project.

\section{References}

[1] M. Naghavi, A. A. Abajobir, C. Abbafati et al., "Global, regional, and national age-sex specific mortality for 264 causes of death, 1980-2016: a systematic analysis for the Global Burden of Disease Study 2016," The Lancet, vol. 390, no. 10100, pp. 1151-1210, 2017.

[2] M. Dehghan, A. Mente, X. Zhang et al., "Associations of fats and carbohydrate intake with cardiovascular disease and mortality in 18 countries from five continents (PURE): a prospective cohort study," The Lancet, vol. 390, no. 10107, pp. 2050-2062, 2017.

[3] S. Yusuf, S. Rangarajan, K. Teo et al., "Cardiovascular risk and events in 17 low-, middle-, and high-income countries," The New England Journal of Medicine, vol. 371, no. 9, pp. 818-827, 2014.

[4] V. Conti, M. Forte, G. Corbi et al., "Sirtuins: possible clinical implications in cardio and cerebrovascular diseases," Current Drug Targets, vol. 18, no. 4, pp. 473-484, 2017.

[5] Y. Lu, Y. D. Wang, X.y. Wang, H. Chen, Z.j. Cai, and M.x. Xiang, "SIRT3 in cardiovascular diseases: emerging roles and therapeutic implications," International Journal of Cardiology, vol. 220, pp. 700-705, 2016.

[6] R. A. Frye, "Phylogenetic classification of prokaryotic and eukaryotic Sir2-like proteins," Biochemical and Biophysical Research Communications, vol. 273, no. 2, pp. 793-798, 2000.

[7] B. Osborne, N. L. Bentley, M. K. Montgomery, and N. Turner, "The role of mitochondrial sirtuins in health and disease," Free Radical Biology \& Medicine, vol. 100, pp. 164174, 2016.

[8] J. Rine, J. N. Strathern, J. B. Hicks, and I. Herskowitz, "A suppressor of mating-type locus mutations in Saccharomyces cerevisiae: evidence for and identification of cryptic matingtype loci," Genetics, vol. 93, no. 4, pp. 877-901, 1979.

[9] B. Schwer, B. J. North, R. A. Frye, M. Ott, and E. Verdin, "The human silent information regulator (Sir)2 homologue hSIRT3 is a mitochondrial nicotinamide adenine dinucleotidedependent deacetylase," The Journal of Cell Biology, vol. 158, no. 4, pp. 647-657, 2002.

[10] J. Bao, Z. Lu, J. J. Joseph et al., "Characterization of the murine SIRT3 mitochondrial localization sequence and 
comparison of mitochondrial enrichment and deacetylase activity of long and short SIRT3 isoforms," Journal of Cellular Biochemistry, vol. 110, no. 1, pp. 238-247, 2010.

[11] M. B. Scher, A. Vaquero, and D. Reinberg, "SirT3 is a nuclear $\mathrm{NAD}^{+}$-dependent histone deacetylase that translocates to the mitochondria upon cellular stress," Genes \& Development, vol. 21, no. 8, pp. 920-928, 2007.

[12] H. M. Cooper and J. N. Spelbrink, "The human SIRT3 protein deacetylase is exclusively mitochondrial," Biochemical Journal, vol. 411, no. 2, pp. 279-285, 2008.

[13] C. Peng, Z. Lu, Z. Xie et al., "The first identification of lysine malonylation substrates and its regulatory enzyme," Mol Cell Proteomics, vol. 10, no. 12, article M111 012658, 2011.

[14] M. J. Rardin, J. C. Newman, J. M. Held et al., "Label-free quantitative proteomics of the lysine acetylome in mitochondria identifies substrates of SIRT3 in metabolic pathways," Proceedings of the National Academy of Sciences of the United States of America, vol. 110, no. 16, pp. 6601-6606, 2013.

[15] D. B. Lombard, F. W. Alt, H. L. Cheng et al., "Mammalian Sir2 homolog SIRT3 regulates global mitochondrial lysine acetylation," Molecular and Cellular Biology, vol. 27, no. 24, pp. 8807-8814, 2007.

[16] H. Bugger, C. N. Witt, and C. Bode, "Mitochondrial sirtuins in the heart," Heart Failure Reviews, vol. 21, no. 5, pp. 519$528,2016$.

[17] N. R. Sundaresan, M. Gupta, G. Kim, S. B. Rajamohan, A. Isbatan, and M. P. Gupta, "Sirt3 blocks the cardiac hypertrophic response by augmenting Foxo3a-dependent antioxidant defense mechanisms in mice," The Journal of Clinical Investigation, vol. 119, no. 9, pp. 2758-2771, 2009.

[18] C. Koentges, K. Pfeil, M. Meyer-Steenbuck et al., "Preserved recovery of cardiac function following ischemia-reperfusion in mice lacking SIRT3," Canadian Journal of Physiology and Pharmacology, vol. 94, no. 1, pp. 72-80, 2016.

[19] C. Koentges, K. Pfeil, T. Schnick et al., "SIRT3 deficiency impairs mitochondrial and contractile function in the heart," Basic Res Cardiol, vol. 110, no. 4, p. 36, 2015.

[20] O. A. Alrob, S. Sankaralingam, C. Ma et al., "Obesity-induced lysine acetylation increases cardiac fatty acid oxidation and impairs insulin signalling," Cardiovascular Research, vol. 103, no. 4, pp. 485-497, 2014.

[21] J. M. Chinawa and A. T. Chinawa, "Assessment of primary health care in a rural health centre in Enugu South East Nigeria," Pakistan Journal of Medical Sciences, vol. 31, no. 1, pp. 60-64, 2015.

[22] G. A. Porter, W. R. Urciuoli, P. S. Brookes, and S. M. Nadtochiy, "SIRT3 deficiency exacerbates ischemia-reperfusion injury: implication for aged hearts," American Journal of Physiology Heart and Circulatory Physiology, vol. 306, no. 12, pp. H1602-H1609, 2014.

[23] K. G. Cheung, L. K. Cole, B. Xiang et al., "Sirtuin-3 (SIRT3) protein attenuates doxorubicin-induced oxidative stress and improves mitochondrial respiration in $\mathrm{H} 9 \mathrm{c} 2$ cardiomyocytes," The Journal of Biological Chemistry, vol. 290, no. 17, pp. 10981-10993, 2015.

[24] H. Zeng, V. R. Vaka, X. He, G. W. Booz, and J. X. Chen, "High-fat diet induces cardiac remodelling and dysfunction: assessment of the role played by SIRT3 loss," Journal of Cellular and Molecular Medicine, vol. 19, no. 8, pp. 18471856, 2015.
[25] V. B. Pillai, N. R. Sundaresan, G. Kim et al., "Exogenous NAD blocks cardiac hypertrophic response via activation of the SIRT3-LKB1-AMP-activated kinase pathway," The Journal of Biological Chemistry, vol. 285, no. 5, pp. 3133-3144, 2010.

[26] W. C. Stanley, F. A. Recchia, and G. D. Lopaschuk, "Myocardial substrate metabolism in the normal and failing heart," Physiological Reviews, vol. 85, no. 3, pp. 1093-1129, 2005.

[27] L. Wei, Y. Zhou, Q. Dai et al., "Oroxylin A induces dissociation of hexokinase II from the mitochondria and inhibits glycolysis by SIRT3-mediated deacetylation of cyclophilin D in breast carcinoma," Cell Death \& Disease, vol. 4, no. 4, article e601, 2013.

[28] P. L. Pedersen, S. Mathupala, A. Rempel, J. F. Geschwind, and Y. H. Ko, "Mitochondrial bound type II hexokinase: a key player in the growth and survival of many cancers and an ideal prospect for therapeutic intervention," Biochimica et Biophysica Acta (BBA) - Bioenergetics, vol. 1555, no. 1-3, pp. 14-20, 2002.

[29] L. W. S. Finley, A. Carracedo, J. Lee et al., "SIRT3 opposes reprogramming of cancer cell metabolism through HIF1 $\alpha$ destabilization," Cancer Cell, vol. 19, no. 3, pp. 416-428, 2011.

[30] R. Paulin, P. Dromparis, G. Sutendra et al., "Sirtuin 3 deficiency is associated with inhibited mitochondrial function and pulmonary arterial hypertension in rodents and humans," Cell Metabolism, vol. 20, no. 5, pp. 827-839, 2014.

[31] M. D. Hirschey, T. Shimazu, E. Goetzman et al., "SIRT3 regulates mitochondrial fatty-acid oxidation by reversible enzyme deacetylation," Nature, vol. 464, no. 7285 , pp. 121125, 2010.

[32] W. Yang, K. Nagasawa, C. Münch et al., "Mitochondrial sirtuin network reveals dynamic SIRT3-dependent deacetylation in response to membrane depolarization," Cell, vol. 167, no. 4, pp. 985-1000.e21, 2016.

[33] B. Schwer, J. Bunkenborg, R. O. Verdin, J. S. Andersen, and E. Verdin, "Reversible lysine acetylation controls the activity of the mitochondrial enzyme acetyl-CoA synthetase 2," Proceedings of the National Academy of Sciences of the United States of America, vol. 103, no. 27, pp. 10224-10229, 2006.

[34] W. C. Hallows, S. Lee, and J. M. Denu, "Sirtuins deacetylate and activate mammalian acetyl-CoA synthetases," Proceedings of the National Academy of Sciences of the United States of America, vol. 103, no. 27, pp. 10230-10235, 2006.

[35] T. Shimazu, M. D. Hirschey, L. Hua et al., "SIRT3 deacetylates mitochondrial 3-hydroxy-3-methylglutaryl CoA synthase 2 and regulates ketone body production," Cell Metabolism, vol. 12, no. 6, pp. 654-661, 2010.

[36] W. Yi, X. Xie, M. Y. Du et al., "Green tea polyphenols ameliorate the early renal damage induced by a high-fat diet via ketogenesis/SIRT3 pathway," Oxidative Medicine and Cellular Longevity, vol. 2017, Article ID 9032792, 14 pages, 2017.

[37] W. C. Hallows, W. Yu, B. C. Smith et al., "Sirt3 promotes the urea cycle and fatty acid oxidation during dietary restriction," Molecular Cell, vol. 41, no. 2, pp. 139-149, 2011.

[38] Y. Yang, H. Cimen, M. J. Han et al., "NAD"-dependent deacetylase SIRT3 regulates mitochondrial protein synthesis by deacetylation of the ribosomal protein MRPL10," The Journal of Biological Chemistry, vol. 285, no. 10, pp. 74177429, 2010.

[39] L. Zhong and R. Mostoslavsky, "Fine tuning our cellular factories: sirtuins in mitochondrial biology," Cell Metabolism, vol. 13, no. 6, pp. 621-626, 2011. 
[40] B.-H. Ahn, H.-S. Kim, S. Song et al., "A role for the mitochondrial deacetylase Sirt3 in regulating energy homeostasis," Proceedings of the National Academy of Sciences of the United States of America, vol. 105, no. 38, pp. 1444714452, 2008.

[41] L. W. S. Finley, W. Haas, V. Desquiret-Dumas et al., "Succinate dehydrogenase is a direct target of sirtuin 3 deacetylase activity," PLoS One, vol. 6, no. 8, article e23295, 2011.

[42] H. Cimen, M.-J. Han, Y. Yang, Q. Tong, H. Koc, and E. C. Koc, "Regulation of succinate dehydrogenase activity by SIRT3 in mammalian mitochondria," Biochemistry, vol. 49, no. 2, pp. 304-311, 2010.

[43] J. Bao, I. Scott, Z. Lu et al., "SIRT3 is regulated by nutrient excess and modulates hepatic susceptibility to lipotoxicity," Free Radical Biology \& Medicine, vol. 49, no. 7, pp. 12301237, 2010.

[44] S. Someya, W. Yu, W. C. Hallows et al., "Sirt3 mediates reduction of oxidative damage and prevention of agerelated hearing loss under caloric restriction," Cell, vol. 143, no. 5, pp. 802-812, 2010.

[45] A. V. Hafner, J. Dai, A. P. Gomes et al., "Regulation of the mPTP by SIRT3-mediated deacetylation of CypD at lysine 166 suppresses age-related cardiac hypertrophy," Aging, vol. 2, no. 12, pp. 914-923, 2010.

[46] E. S. Park, J. C. Kang, Y. C. Jang et al., "Cardioprotective effects of rhamnetin in H9c2 cardiomyoblast cells under $\mathrm{H}_{2} \mathrm{O}_{2}$-induced apoptosis," Journal of Ethnopharmacology, vol. 153 , no. 3, pp. 552-560, 2014.

[47] V. B. Pillai, N. R. Sundaresan, and M. P. Gupta, "Regulation of Akt signaling by sirtuins its implication in cardiac hypertrophy and aging," Circulation Research, vol. 114, no. 2, pp. 368-378, 2014.

[48] C. Dong, D. Della-Morte, L. Wang et al., "Association of the sirtuin and mitochondrial uncoupling protein genes with carotid plaque," PLoS One, vol. 6, no. 11, article e27157, 2011.

[49] L. Li, H. Zeng, X. Hou, X. He, and J. X. Chen, "Myocardial injection of apelin-overexpressing bone marrow cells improves cardiac repair via upregulation of Sirt3 after myocardial infarction," PLoS One, vol. 8, no. 9, 2013.

[50] A. Giralt, E. Hondares, J. A. Villena et al., "Peroxisome proliferator-activated receptor- $\gamma$ coactivator- $1 \alpha$ controls transcription of the Sirt3 gene, an essential component of the thermogenic brown adipocyte phenotype," The Journal of Biological Chemistry, vol. 286, no. 19, pp. 16958-16966, 2011.

[51] O. M. Palacios, J. J. Carmona, S. Michan et al., "Diet and exercise signals regulate SIRT3 and activate AMPK and PGC- $1 \alpha$ in skeletal muscle," Aging, vol. 1, no. 9, pp. 771-783, 2009.

[52] M. D. Hirschey, T. Shimazu, E. Jing et al., "SIRT3 deficiency and mitochondrial protein hyperacetylation accelerate the development of the metabolic syndrome," Molecular Cell, vol. 44, no. 2, pp. 177-190, 2011.

[53] R. Tao, M. C. Coleman, J. D. Pennington et al., "Sirt3-mediated deacetylation of evolutionarily conserved lysine 122 regulates MnSOD activity in response to stress," Molecular Cell, vol. 40, no. 6, pp. 893-904, 2010.

[54] X. Qiu, K. Brown, M. D. Hirschey, E. Verdin, and D. Chen, "Calorie restriction reduces oxidative stress by SIRT3mediated SOD2 activation," Cell Metabolism, vol. 12, no. 6, pp. 662-667, 2010.
[55] A. E. Dikalova, H. A. Itani, R. R. Nazarewicz et al., "Sirt3 impairment and SOD2 hyperacetylation in vascular oxidative stress and hypertension," Circulation Research, vol. 121, no. 5, pp. 564-574, 2017.

[56] X. Xie, L. Wang, B. Zhao, Y. Chen, and J. Li, "SIRT3 mediates decrease of oxidative damage and prevention of ageing in porcine fetal fibroblasts," Life Sciences, vol. 177, pp. 41-48, 2017.

[57] R. A. H. van de Ven, D. Santos, and M. C. Haigis, "Mitochondrial sirtuins and molecular mechanisms of aging," Trends in Molecular Medicine, vol. 23, no. 4, pp. 320-331, 2017.

[58] N. R. Sundaresan, S. A. Samant, V. B. Pillai, S. B. Rajamohan, and M. P. Gupta, "SIRT3 is a stress-responsive deacetylase in cardiomyocytes that protects cells from stress-mediated cell death by deacetylation of Ku70," Molecular and Cellular Biology, vol. 28, no. 20, pp. 6384-6401, 2008.

[59] A. Signorile, A. Santeramo, G. Tamma et al., "Mitochondrial cAMP prevents apoptosis modulating Sirt3 protein level and OPA1 processing in cardiac myoblast cells," Biochimica et Biophysica Acta-Molecular Cell Research, vol. 1864, no. 2, pp. 355-366, 2017.

[60] S. A. Samant, H. J. Zhang, Z. Hong et al., "SIRT3 deacetylates and activates OPA1 to regulate mitochondrial dynamics during stress," Molecular and Cellular Biology, vol. 34, no. 5, pp. 807-819, 2014.

[61] A. Cheng, Y. Yang, Y. Zhou et al., "Mitochondrial SIRT3 mediates adaptive responses of neurons to exercise and metabolic and excitatory challenges," Cell Metabolism, vol. 23, no. 1, pp. 128-142, 2016.

[62] T. Bochaton, C. Crola-Da-Silva, B. Pillot et al., "Inhibition of myocardial reperfusion injury by ischemic postconditioning requires sirtuin 3-mediated deacetylation of cyclophilin D," Journal of Molecular and Cellular Cardiology, vol. 84, pp. 61-69, 2015.

[63] R. M. Bell and D. M. Yellon, "There is more to life than revascularization: therapeutic targeting of myocardial ischemia/ reperfusion injury," Cardiovascular Therapeutics, vol. 29, no. 6, pp. e67-e79, 2011.

[64] R. M. Parodi-Rullán, X. Chapa-Dubocq, P. J. Rullan, S. Jang, and S. Javadov, "High sensitivity of SIRT3 deficient hearts to ischemia-reperfusion is associated with mitochondrial abnormalities," Frontiers in Pharmacology, vol. 8, p. 275, 2017.

[65] X. He, H. Zeng, and J.-X. Chen, "Ablation of SIRT3 causes coronary microvascular dysfunction and impairs cardiac recovery post myocardial ischemia," International Journal of Cardiology, vol. 215, pp. 349-357, 2016.

[66] M. S. Klishadi, F. Zarei, S. H. Hejazian, A. Moradi, M. Hemati, and F. Safari, "Losartan protects the heart against ischemia reperfusion injury: sirtuin3 involvement," Journal of Pharmacy \& Pharmaceutical Sciences, vol. 18, no. 1, pp. 112123, 2015.

[67] K. M. Baker, G. W. Booz, and D. E. Dostal, "Cardiac actions of angiotensin II: role of an intracardiac renin-angiotensin system," Annual Review of Physiology, vol. 54, no. 1, pp. 227-241, 1992.

[68] B. C. Yang, M. I. Phillips, P. E. J. Ambuehl, L. P. Shen, P. Mehta, and J. L. Mehta, "Increase in angiotensin II type 1 receptor expression immediately after ischemia-reperfusion in isolated rat hearts," Circulation, vol. 96, no. 3, pp. 922 926, 1997. 
[69] A. Benigni, D. Corna, C. Zoja et al., "Disruption of the Ang II type 1 receptor promotes longevity in mice," The Journal of Clinical Investigation, vol. 119, no. 3, pp. 524-530, 2009.

[70] R. Parodi-Rullan, G. Barreto-Torres, L. Ruiz, J. Casasnovas, and S. Javadov, "Direct renin inhibition exerts an antihypertrophic effect associated with improved mitochondrial function in post-infarction heart failure in diabetic rats," Cellular Physiology and Biochemistry, vol. 29, no. 5-6, pp. 841850, 2012.

[71] M. Chen, X. Zhu, L. Ran, H. Lang, L. Yi, and M. Mi, "Trimethylamine-N-oxide induces vascular inflammation by activating the NLRP3 inflammasome through the SIRT3SOD2-mtROS signaling pathway," Journal of the American Heart Association, vol. 6, no. 9, article e006347, 2017.

[72] H. K. Jiang, Y. Miao, Y. H. Wang et al., "Aerobic interval training protects against myocardial infarction-induced oxidative injury by enhancing antioxidase system and mitochondrial biosynthesis," Clinical and Experimental Pharmacology and Physiology, vol. 41, no. 3, pp. 192-201, 2014.

[73] S. Winnik, D. S. Gaul, F. Preitner et al., "Deletion of Sirt3 does not affect atherosclerosis but accelerates weight gain and impairs rapid metabolic adaptation in LDL receptor knockout mice: implications for cardiovascular risk factor development," Basic Research in Cardiology, vol. 109, no. 1, p. 399, 2014.

[74] N. Frey, H. A. Katus, E. N. Olson, and J. A. Hill, "Hypertrophy of the heart: a new therapeutic target?," Circulation, vol. 109, no. 13, pp. 1580-1589, 2004.

[75] N. R. Sundaresan, S. Bindu, V. B. Pillai et al., "SIRT3 blocks aging-associated tissue fibrosis in mice by deacetylating and activating glycogen synthase kinase $3 \beta$," Molecular and Cellular Biology, vol. 36, no. 5, pp. 678-692, 2015.

[76] V. B. Pillai, S. Samant, N. R. Sundaresan et al., "Honokiol blocks and reverses cardiac hypertrophy in mice by activating mitochondrial Sirt3," Nature Communications, vol. 6, p. 6656, 2015.

[77] T. Chen, J. Liu, N. Li et al., "Mouse SIRT3 attenuates hypertrophy-related lipid accumulation in the heart through the deacetylation of LCAD," PLoS One, vol. 10, no. 3, article e0118909, 2015.

[78] J. M. Grillon, K. R. Johnson, K. Kotlo, and R. S. Danziger, "Non-histone lysine acetylated proteins in heart failure," Biochimica et Biophysica Acta (BBA) - Molecular Basis of Disease, vol. 1822, no. 4, pp. 607-614, 2012.

[79] L. Yu, B. Gong, W. Duan et al., "Melatonin ameliorates myocardial ischemia/reperfusion injury in type 1 diabetic rats by preserving mitochondrial function: role of AMPK-PGC- $1 \alpha$ SIRT3 signaling," Scientific Reports, vol. 7, article 41337, 2017.

[80] P. Pacher and C. Szabo, "Role of poly(ADP-ribose) polymerase 1 (PARP-1) in cardiovascular diseases: the therapeutic potential of PARP inhibitors," Cardiovascular Drug Reviews, vol. 25, no. 3, pp. 235-260, 2007.

[81] H. Yang, T. Yang, J. A. Baur et al., "Nutrient-sensitive mitochondrial $\mathrm{NAD}^{+}$levels dictate cell survival," Cell, vol. 130, no. 6, pp. 1095-1107, 2007.

[82] J. You, Z. Yue, S. Chen et al., "Receptor-interacting protein 140 represses sirtuin 3 to facilitate hypertrophy, mitochondrial dysfunction and energy metabolic dysfunction in cardiomyocytes," Acta Physiologica, vol. 220, no. 1, pp. 58-71, 2017.
[83] A. Nikiforov, C. Dolle, M. Niere, and M. Ziegler, "Pathways and subcellular compartmentation of NAD biosynthesis in human cells: from entry of extracellular precursors to mitochondrial NAD generation," The Journal of Biological Chemistry, vol. 286, no. 24, pp. 21767-21778, 2011.

[84] Z. Yue, Y. Ma, J. You et al., "NMNAT3 is involved in the protective effect of SIRT3 in Ang II-induced cardiac hypertrophy," Experimental Cell Research, vol. 347, no. 2, pp. 261-273, 2016.

[85] X. Tang, X.-F. Chen, H.-Z. Chen, and D.-P. Liu, "Mitochondrial sirtuins in cardiometabolic diseases," Clinical Science, vol. 131, no. 16, pp. 2063-2078, 2017.

[86] Y. C. Lai, D. M. Tabima, J. J. Dube et al., "SIRT3-AMP-activated protein kinase activation by nitrite and metformin improves hyperglycemia and normalizes pulmonary hypertension associated with heart failure with preserved ejection fraction," Circulation, vol. 133, no. 8, pp. 717-731, 2016.

[87] X. Guo, F. Yan, J. Li, C. Zhang, and P. Bu, "SIRT3 attenuates AngII-induced cardiac fibrosis by inhibiting myofibroblasts transdifferentiation via STAT3-NFATc2 pathway," American Journal of Translational Research, vol. 9, no. 7, pp. 32583269, 2017.

[88] X. Guo, F. Yan, X. Shan et al., "SIRT3 inhibits Ang II-induced transdifferentiation of cardiac fibroblasts through $\beta$-catenin/ PPAR- $\gamma$ signaling," Life Sciences, vol. 186, pp. 111-117, 2017.

[89] T. Wei, G. Huang, J. Gao et al., "Sirtuin 3 deficiency accelerates hypertensive cardiac remodeling by impairing angiogenesis," Journal of the American Heart Association, vol. 6, no. 8, article e006114, 2017.

[90] J. L. Zamorano, P. Lancellotti, D. Rodriguez Muñoz et al., "2016 ESC Position Paper on cancer treatments and cardiovascular toxicity developed under the auspices of the ESC Committee for Practice Guidelines The Task Force for cancer treatments and cardiovascular toxicity of the European Society of Cardiology (ESC)," European Journal of Heart Failure, vol. 19, no. 1, pp. 9-42, 2017.

[91] S. Zhang, X. Liu, T. Bawa-Khalfe et al., "Identification of the molecular basis of doxorubicin-induced cardiotoxicity," Nature Medicine, vol. 18, no. 11, pp. 1639-1642, 2012.

[92] I. Marques-Aleixo, E. Santos-Alves, D. Mariani et al., "Physical exercise prior and during treatment reduces sub-chronic doxorubicin-induced mitochondrial toxicity and oxidative stress," Mitochondrion, vol. 20, pp. 22-33, 2015.

[93] V. B. Pillai, S. Bindu, W. Sharp et al., "Sirt3 protects mitochondrial DNA damage and blocks the development of doxorubicin-induced cardiomyopathy in mice," American Journal of Physiology-Heart and Circulatory Physiology, vol. 310, no. 8, pp. H962-H972, 2016.

[94] V. W. Dolinsky, "The role of sirtuins in mitochondrial function and doxorubicin-induced cardiac dysfunction," Biological Chemistry, vol. 398, no. 9, pp. 955-974, 2017.

[95] Q. Du, B. Zhu, Q. Zhai, and B. Yu, "Sirt3 attenuates doxorubicin-induced cardiac hypertrophy and mitochondrial dysfunction via suppression of Bnip3," American Journal of Translational Research, vol. 9, no. 7, pp. 3360-3373, 2017.

[96] J. B. Lin, S. Kubota, N. Ban et al., "NAMPT-mediated NAD biosynthesis is essential for vision in mice," Cell Reports, vol. 17, no. 1, pp. 69-85, 2016.

[97] Y. Zeng, K. Yang, F. Wang et al., "The glucagon like peptide 1 analogue, exendin-4, attenuates oxidative stress-induced 
retinal cell death in early diabetic rats through promoting Sirt1 and Sirt3 expression," Experimental Eye Research, vol. 151, pp. 203-211, 2016.

[98] S. Balaiya, K. K. Abu-Amero, A. A. Kondkar, and K. V. Chalam, "Sirtuins expression and their role in retinal diseases," Oxidative Medicine and Cellular Longevity, vol. 2017, Article ID 3187594, 11 pages, 2017.

[99] E. Jing, B. Emanuelli, M. D. Hirschey et al., "Sirtuin-3 (Sirt3) regulates skeletal muscle metabolism and insulin signaling via altered mitochondrial oxidation and reactive oxygen species production," Proceedings of the National Academy of Sciences of the United States of America, vol. 108, no. 35, pp. 14608-14613, 2011.

[100] Y. Guan, Z.-J. Cui, B. Sun, L.-P. Han, C.-J. Li, and L.-M. Chen, "Celastrol attenuates oxidative stress in the skeletal muscle of diabetic rats by regulating the AMPK-PGC1 $\alpha$-SIRT3 signaling pathway," International Journal of Molecular Medicine, vol. 37, no. 5, pp. 1229-1238, 2016.

[101] L. Shi, T. Zhang, Y. Zhou et al., "Dihydromyricetin improves skeletal muscle insulin sensitivity by inducing autophagy via the AMPK-PGC- $1 \alpha$-Sirt3 signaling pathway," Endocrine, vol. 50, no. 2, pp. 378-389, 2015.

[102] W. Yu, B. Gao, N. Li et al., "Sirt3 deficiency exacerbates diabetic cardiac dysfunction: role of Foxo3A-Parkin-mediated mitophagy," Biochimica et Biophysica Acta-Molecular Basis of Disease, vol. 1863, no. 8, pp. 1973-1983, 2017.

[103] X. Hou, H. Zeng, X. He, and J.-X. Chen, "Sirt3 is essential for apelin-induced angiogenesis in post-myocardial infarction of diabetes," Journal of Cellular and Molecular Medicine, vol. 19, no. 1, pp. 53-61, 2015.

[104] M. Zhang, J. Lin, S. Wang et al., "Melatonin protects against diabetic cardiomyopathy through Mst1/Sirt3 signaling," Journal of Pineal Research, vol. 63, no. 2, 2017.

[105] M. R. Sultana, P. K. Bagul, P. B. Katare, S. Anwar Mohammed, R. Padiya, and S. K. Banerjee, "Garlic activates SIRT-3 to prevent cardiac oxidative stress and mitochondrial dysfunction in diabetes," Life Sciences, vol. 164, pp. 42-51, 2016.

[106] S. S. Vadvalkar, S. Matsuzaki, C. A. Eyster et al., "Decreased mitochondrial pyruvate transport activity in the diabetic heart role of mitochondrial pyruvate carrier 2 (MPC2) acetylation," The Journal of Biological Chemistry, vol. 292, no. 11, pp. 4423-4433, 2017.

[107] A. Faria and S. J. Persaud, "Cardiac oxidative stress in diabetes: mechanisms and therapeutic potential," Pharmacology \& Therapeutics, vol. 172, pp. 50-62, 2017.

[108] W. C. Stanley, E. R. Dabkowski, R. F. Ribeiro, and K. A. O'Connell, "Dietary fat and heart failure: moving from lipotoxicity to lipoprotection," Circulation Research, vol. 110, no. 5, pp. 764-776, 2012.

[109] Y. Zhou, A. C. K. Chung, R. Fan et al., "Sirt3 deficiency increased the vulnerability of pancreatic beta cells to oxidative stress-induced dysfunction," Antioxidants \& Redox Signaling, vol. 27, no. 13, pp. 962-976, 2017.

[110] M. H. Forouzanfar, A. Afshin, L. T. Alexander et al., "Global, regional, and national comparative risk assessment of 79 behavioural, environmental and occupational, and metabolic risks or clusters of risks, 1990-2015: a systematic analysis for the Global Burden of Disease Study 2015," The Lancet, vol. 388, no. 10063, pp. 1659-1724, 2016.

[111] G. B. Waypa, S. W. Osborne, J. D. Marks, S. K. Berkelhamer, J. Kondapalli, and P. T. Schumacker, "Sirtuin 3 deficiency does not augment hypoxia-induced pulmonary hypertension," American Journal of Respiratory Cell and Molecular Biology, vol. 49, no. 6, pp. 885-891, 2013.

[112] N. Trevino-Saldana and G. Garcia-Rivas, "Regulation of sirtuin-mediated protein deacetylation by cardioprotective phytochemicals," Oxidative Medicine and Cellular Longevity, vol. 2017, Article ID 1750306, 16 pages, 2017.

[113] B. N. M. Zordoky, I. M. Robertson, and J. R. B. Dyck, "Preclinical and clinical evidence for the role of resveratrol in the treatment of cardiovascular diseases," Biochimica et Biophysica Acta-Molecular Basis of Disease, vol. 1852, no. 6, pp. 1155-1177, 2015.

[114] G. Han, J. Xia, J. Gao, Y. Inagaki, W. Tang, and N. Kokudo, "Anti-tumor effects and cellular mechanisms of resveratrol," Drug Discoveries \& Therapeutics, vol. 9, no. 1, pp. 1-12, 2015.

[115] T. Chen, J. Li, J. Liu et al., "Activation of SIRT3 by resveratrol ameliorates cardiac fibrosis and improves cardiac function via the TGF- $\beta /$ Smad3 pathway," American Journal of Physiology-Heart and Circulatory Physiology, vol. 308, no. 5, pp. H424-H434, 2015.

[116] M. Zhang, Z. Zhao, M. Shen et al., "Polydatin protects cardiomyocytes against myocardial infarction injury by activating Sirt3," Biochimica et Biophysica Acta (BBA) - Molecular Basis of Disease, vol. 1863, no. 8, pp. 1962-1972, 2017.

[117] A. R. Coelho, T. R. Martins, R. Couto et al., "Berberineinduced cardioprotection and Sirt3 modulation in doxorubicintreated H9c2 cardiomyoblasts," Biochimica et Biophysica Acta (BBA) - Molecular Basis of Disease, vol. 1863, no. 11, pp. 2904-2923, 2017.

[118] V. B. Pillai, A. Kanwal, Y. H. Fang et al., "Honokiol, an activator of Sirtuin-3 (SIRT3) preserves mitochondria and protects the heart from doxorubicin-induced cardiomyopathy in mice," Oncotarget, vol. 8, no. 21, pp. 34082-34098, 2017.

[119] C. Zhao, T. Sakaguchi, K. Fujita et al., "Pomegranate-derived polyphenols reduce reactive oxygen species production via SIRT3-mediated SOD2 activation," Oxidative Medicine and Cellular Longevity, vol. 2016, Article ID 2927131, 9 pages, 2016.

[120] N. Hu, J. Ren, and Y. Zhang, "Mitochondrial aldehyde dehydrogenase obliterates insulin resistance-induced cardiac dysfunction through deacetylation of PGC-1 $\alpha$," Oncotarget, vol. 7, no. 47, pp. 76398-76414, 2016.

[121] J. Liu, Y. Tang, Z. Feng et al., "Acetylated FoxO1 mediates high-glucose induced autophagy in $\mathrm{H} 9 \mathrm{c} 2$ cardiomyoblasts: regulation by a polyphenol -(-)-epigallocatechin-3-gallate," Metabolism-Clinical and Experimental, vol. 63, no. 10, pp. 1314-1323, 2014.

[122] J. Xiao, X. Sheng, X. Zhang, M. Guo, and X. Ji, "Curcumin protects against myocardial infarction-induced cardiac fibrosis via SIRT1 activation in vivo and in vitro," Drug Design Development and Therapy, vol. 10, pp. 1267-1277, 2016.

[123] H. Tronchere, M. Cinato, A. Timotin et al., "Inhibition of PIKfyve prevents myocardial apoptosis and hypertrophy through activation of SIRT3 in obese mice," EMBO Molecular Medicine, vol. 9, no. 6, pp. 770-785, 2017.

[124] M. Zhai, B. Li, W. Duan et al., "Melatonin ameliorates myocardial ischemia reperfusion injury through SIRT3dependent regulation of oxidative stress and apoptosis," Journal of Pineal Research, vol. 63, no. 2, 2017.

[125] J. Hu, L. Zhang, Y. Yang et al., "Melatonin alleviates postinfarction cardiac remodeling and dysfunction by inhibiting Mst1," Journal of Pineal Research, vol. 62, no. 1, 2017. 
[126] W. Xia and K. Geng, "A sirtuin activator and an antiinflammatory molecule-multifaceted roles of adjudin and its potential applications for aging-related diseases," Seminars in Cell \& Developmental Biology, vol. 59, pp. 71-78, 2016.

[127] F. Yang, L. Zhou, D. Wang, Z. Wang, and Q. Y. Huang, "Minocycline ameliorates hypoxia-induced blood-brain barrier damage by inhibition of HIF- $1 \alpha$ through SIRT-3/PHD- 2 degradation pathway," Neuroscience, vol. 304, pp. 250-259, 2015.

[128] M. Kobayashi, K. Takeda, T. Narita et al., "Mitochondrial intermediate peptidase is a novel regulator of sirtuin-3 activation by caloric restriction," FEBS Letters, vol. 591, no. 24, pp. 4067-4073, 2017.

[129] N. Diaz-Morales, S. Rovira-Llopis, C. Bañuls et al., "Does metformin protect diabetic patients from oxidative stress and leukocyte-endothelium interactions?," Antioxidants \& Redox Signaling, vol. 27, no. 17, pp. 1439-1445, 2017.

[130] R. H. Houtkooper and J. Auwerx, "Exploring the therapeutic space around NAD ," Journal of Cell Biology, vol. 199, no. 2, pp. 205-209, 2012.

[131] J. Yoshino, K. F. Mills, M. J. Yoon, and S. Imai, “Nicotinamide mononucleotide, a key $\mathrm{NAD}^{+}$intermediate, treats the pathophysiology of diet- and age-induced diabetes in mice," Cell Metabolism, vol. 14, no. 4, pp. 528-536, 2011.

[132] W. T. Harkcom, A. K. Ghosh, M. S. Sung et al., "NAD ${ }^{+}$and SIRT3 control microtubule dynamics and reduce susceptibility to antimicrotubule agents," Proceedings of the National Academy of Sciences of the United States of America, vol. 111, no. 24, pp. E2443-E2452, 2014.

[133] A. P. Gomes, N. L. Price, A. J. Y. Ling et al., "Declining $\mathrm{NAD}^{+}$induces a pseudohypoxic state disrupting nuclearmitochondrial communication during aging," Cell, vol. 155, no. 7, pp. 1624-1638, 2013.

[134] K. D. Brown, S. Maqsood, J. Y. Huang et al., "Activation of SIRT3 by the $\mathrm{NAD}^{+}$precursor nicotinamide riboside protects from noise-induced hearing loss," Cell Metabolism, vol. 20, no. 6, pp. 1059-1068, 2014.

[135] B. R. Sabari, D. Zhang, C. D. Allis, and Y. Zhao, "Metabolic regulation of gene expression through histone acylations," Nature Reviews Molecular Cell Biology, vol. 18, no. 2, pp. 90-101, 2017.

[136] M. Gertz and C. Steegborn, "Using mitochondrial sirtuins as drug targets: disease implications and available compounds," Cellular and Molecular Life Sciences, vol. 73, no. 15, pp. 28712896, 2016.

[137] C. Schölz, B. T. Weinert, S. A. Wagner et al., "Acetylation site specificities of lysine deacetylase inhibitors in human cells," Nature Biotechnology, vol. 33, no. 4, pp. 415-423, 2015.

[138] J. Lu, H. Zhang, X. Chen et al., "A small molecule activator of SIRT3 promotes deacetylation and activation of manganese superoxide dismutase," Free Radical Biology \& Medicine, vol. 112, pp. 287-297, 2017.

[139] Y. H. Jung, H. J. Lee, J. S. Kim, S. J. Lee, and H. J. Han, “EphB2 signaling-mediated Sirt3 expression reduces MSC senescence by maintaining mitochondrial ROS homeostasis," Free Radical Biology \& Medicine, vol. 110, pp. 368-380, 2017.

[140] Y. Chen, C. Chen, B. Dong et al., "AMPK attenuates ventricular remodeling and dysfunction following aortic banding in mice via the Sirt3/oxidative stress pathway," European Journal of Pharmacology, vol. 814, pp. 335-342, 2017.

[141] V. T. Vachharajani, T. Liu, X. Wang, J. J. Hoth, B. K. Yoza, and C. E. McCall, "Sirtuins link inflammation and metabolism," Journal of Immunology Research, vol. 2016, Article ID 8167273, 10 pages, 2016.

[142] P. Cheng, W. Zeng, L. Li et al., "PLGA-PNIPAM microspheres loaded with the gastrointestinal nutrient $\mathrm{NaB}$ ameliorate cardiac dysfunction by activating Sirt3 in acute myocardial infarction," Advanced Science, vol. 3, no. 12, article 1600254, 2016.

[143] S. P. Singh, J. Schragenheim, J. Cao, J. R. Falck, N. G. Abraham, and L. Bellner, "PGC-1 alpha regulates HO-1 expression, mitochondrial dynamics and biogenesis: role of epoxyeicosatrienoic acid," Prostaglandins \& Other Lipid Mediators, vol. 125, pp. 8-18, 2016.

[144] W. Sun, L. Zhao, X. Song et al., "MicroRNA-210 modulates the cellular energy metabolism shift during $\mathrm{H}_{2} \mathrm{O}_{2}$-induced oxidative stress by repressing ISCU in H9c2 cardiomyocytes," Cellular Physiology and Biochemistry, vol. 43, no. 1, pp. 383394, 2017.

[145] X. Chen and M. Rosbash, "MicroRNA-92a is a circadian modulator of neuronal excitability in Drosophila," Nature Communications, vol. 8, article 14707, Article ID 28276426, 2017.

[146] R. C. Poulsen, H. J. Knowles, A. J. Carr, and P. A. Hulley, "Cell differentiation versus cell death: extracellular glucose is a key determinant of cell fate following oxidative stress exposure," Cell Death \& Disease, vol. 5, no. 2, article e1074, 2014.

[147] Y. Cheng, J. Mai, T. Hou, and J. Ping, "MicroRNA-421 induces hepatic mitochondrial dysfunction in non-alcoholic fatty liver disease mice by inhibiting sirtuin 3," Biochemical and Biophysical Research Communications, vol. 474, no. 1, pp. $57-63,2016$. 


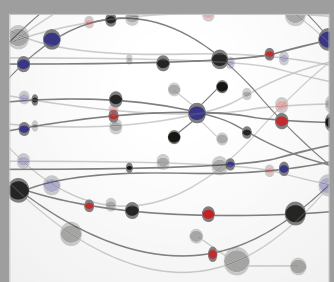

The Scientific World Journal
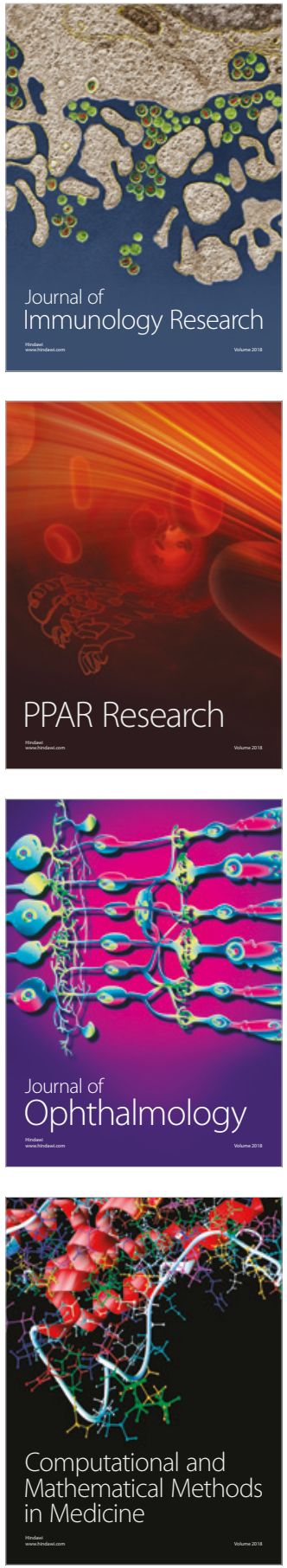

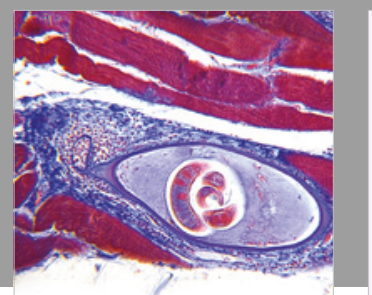

Gastroenterology Research and Practice

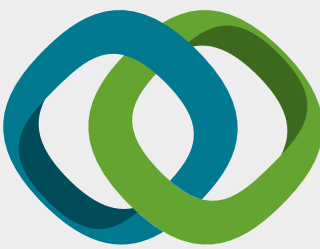

\section{Hindawi}

Submit your manuscripts at

www.hindawi.com
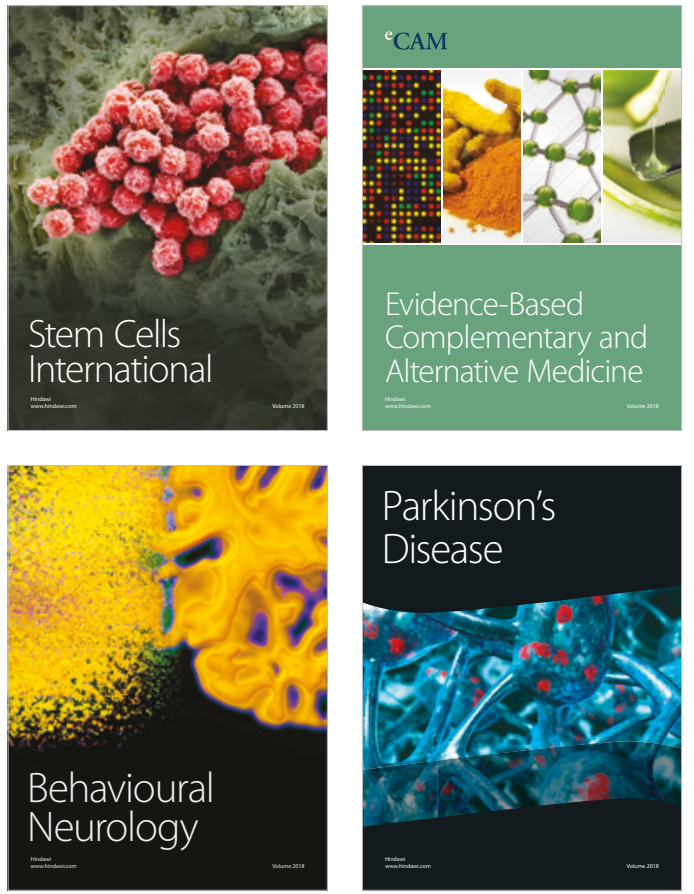

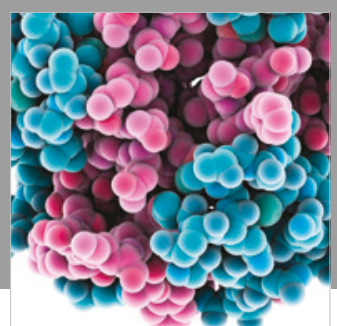

ournal of

Diabetes Research

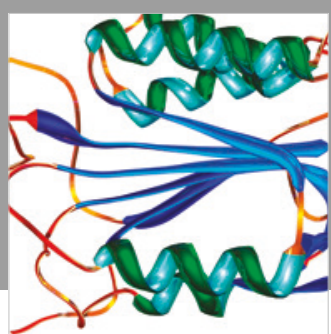

Disease Markers
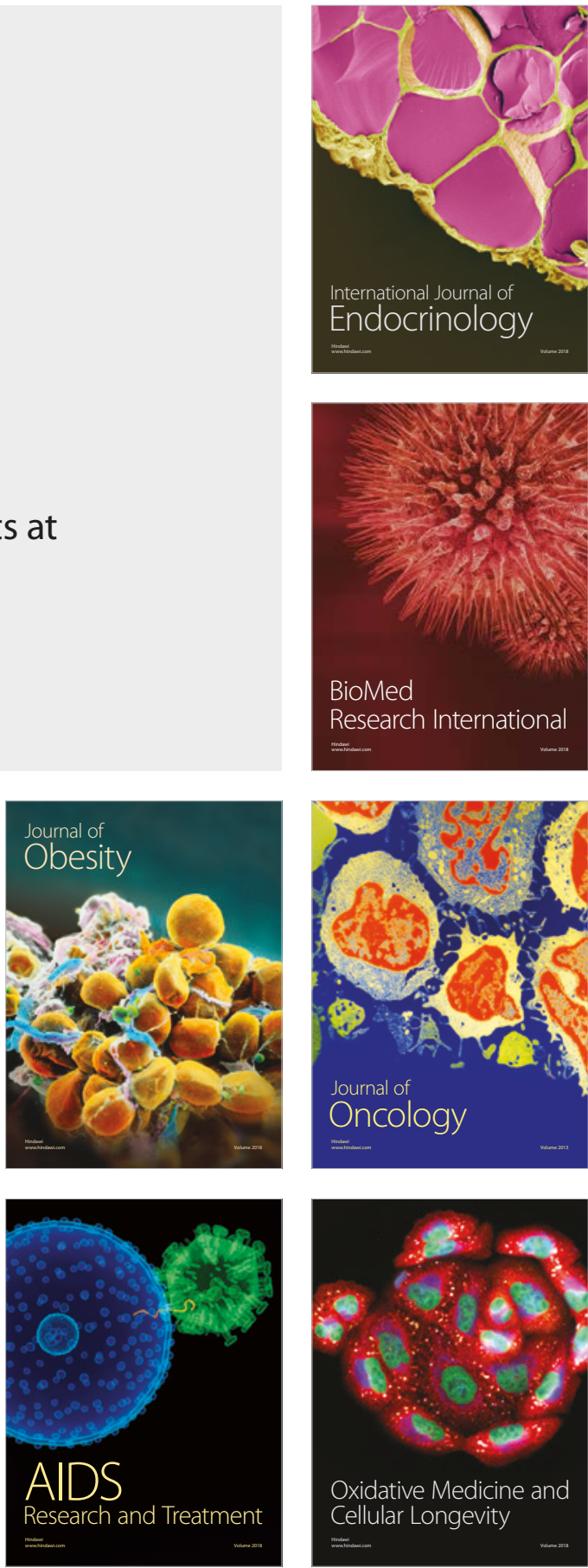